Full length article

\title{
Frequent binge drinking five to six years after exposure to 9/11: Findings from the World Trade Center Health Registry
}

\author{
Alice E. Welch ${ }^{\mathrm{a}, *}$, Kimberly Caramanica ${ }^{\mathrm{a}}$, Carey B. Maslowa ${ }^{\mathrm{a}}$, James E. Cone ${ }^{\mathrm{a}}$, \\ Mark R. Farfel ${ }^{a}$, Katherine M. Keyes ${ }^{\mathrm{b}, \mathrm{c}}$, Steven D. Stellman ${ }^{\mathrm{a}, \mathrm{b}}$, Deborah S. Hasin ${ }^{\mathrm{b}}$ \\ a New York City Department of Health and Mental Hygiene, 42-09 28th Street, 07-24, Queens, NY 11101, USA \\ b Department of Epidemiology, Mailman School of Public Health, Columbia University, New York, NY 10032, USA \\ c Department of Psychiatry, College of Physicians and Surgeons, Columbia University, New York, NY, 10032, USA
}

\section{A R T I C L E I N F O}

\section{Article history:}

Received 8 January 2014

Received in revised form 16 April 2014

Accepted 17 April 2014

Available online 28 April 2014

\section{Keywords:}

9/11

Frequent binge drinking

World Trade Center

September 11, 2001

Posttraumatic stress disorder

PTSD

\begin{abstract}
A B S T R A C T
Background: Exposure to 9/11 may have considerable long-term impact on health behaviors, including increased alcohol consumption. We examined the association between frequent binge drinking, posttraumatic stress disorder (PTSD), and number of 9/11-specific experiences among World Trade Center Health Registry (Registry) enrollees five-to-six years after 9/11.

Methods: Participants included 41,284 lower Manhattan residents, workers, passers-by, and rescue/recovery workers aged 18 or older without a pre-9/11 PTSD diagnosis who completed Wave 1 (2003-2004) and Wave 2 (2006-2007) interviews. Frequent binge drinking was defined as consuming five or more drinks on five or more occasions in the prior 30 days at Wave 2. Probable PTSD was defined as scoring 44 or greater on the PTSD Checklist. 9/11 exposure was measured as the sum of 12 experiences and grouped as none/low (0-1), medium (2-3), high (4-5) and very high (6+).

Results: Frequent binge drinking was significantly associated with increasing 9/11 exposure and PTSD. Those with very high and high exposures had a higher prevalence of frequent binge drinking (13.7\% and $9.8 \%$, respectively) than those with medium and low exposures ( $7.5 \%$ and $4.4 \%$, respectively). Upon stratification, very high and high exposures were associated with frequent binge drinking in both the PTSD and no PTSD subgroups.

Conclusions: Our findings suggest that 9/11 exposure had an impact on frequent binge drinking fiveto-six years later among Registry enrollees. Understanding the effects of traumatic exposure on alcohol use is important to identify risk factors for post-disaster alcohol misuse, inform policy, and improve post-disaster psychological and alcohol screening and counseling.
\end{abstract}

(c) 2014 Elsevier Ireland Ltd. All rights reserved.

\section{Introduction}

The September 11, 2001 (9/11) terrorist attacks have had considerable long-term consequences for physical and mental health and health behaviors for many of those directly exposed, including Manhattan residents, passers-by, office workers, and rescue and recovery workers (Brackbill et al., 2009). Additionally, 9/11 exposures were associated with high levels of psychological stress on dimensions linked with increased risk for heavy drinking and alcohol problems (Keyes et al., 2011), including severity, unexpectedness, and threat to physical as well as emotional integrity. Indeed, an elevated prevalence of post-disaster alcohol use has been

\footnotetext{
* Corresponding author. Tel.: +1 347396 2856; fax: +1 3473962893.

E-mail address: awelch1@health.nyc.gov (A.E. Welch).
}

consistently described in multiple populations exposed to traumatic events. Higher levels of alcohol use have been found in firefighters who served as rescue workers during the Oklahoma City bombing (North et al., 2002), survivors of Hurricane Katrina (Flory et al., 2009), and individuals who were impacted by the 9/11 terrorist attacks, including New York City (NYC) residents (Vlahov et al., 2002), Pentagon staff (Grieger et al., 2003), members of the Fire Department of New York (FDNY; Berninger et al., 2010) and individuals living in an area adjacent to NYC (Hasin et al., 2007); these findings are summarized in a meta-analysis by DiMaggio et al. (2009).

Among Manhattan residents living south of 110th Street, Vlahov et al. (2004) identified increases in alcohol use associated with level of 9/11-related exposure up to nine months after 9/11. Similarly, among NYC adults, level of exposure on 9/11 was associated with binge drinking one, and again two years after 9/11 (Boscarino et al., 
2006). Personal proximity to the WTC on the day of the attack was associated with subsequent elevated drinking three months post9/11 among residents of an area directly adjacent to NYC in New Jersey, even after controlling for a lifetime history of alcohol dependence (Hasin et al., 2007). Moreover, the prevalence of post-disaster problem drinking was two to six times greater among individuals with symptoms of posttraumatic stress disorder (PTSD) compared to those without symptoms (Adams et al., 2006; Berninger et al., 2010; Boscarino et al., 2011; Vlahov et al., 2006). These findings demonstrate that there likely exists a complex relationship among exposure to the events of 9/11, symptoms of PTSD, and problem drinking behaviors.

Despite multiple studies documenting elevated rates of binge drinking following exposure to 9/11, several important areas remain unexplored. Studies have usually measured drinking after relatively short periods of time (one month to two years postexposure); less is known about longer-term effects of 9/11 exposures and drinking, or about possible co-factors that may increase risk or extend it across time. Additionally, with the exception of Adams and Boscarino (2005), previous studies had limited data on type and intensity of 9/11 exposures and their relationship to drinking patterns. Very little information is available on how exposure to severe, unexpected trauma among adults impacts longterm drinking patterns. We examined the relationship between intensity of 9/11-related exposures, PTSD, and frequent binge drinking five to six years after $9 / 11$ in a large cohort of adults directly exposed to the events of 9/11.

\section{Methods}

\subsection{Data source}

The World Trade Center Health Registry (the Registry) is a cohort study of 71,434 individuals directly exposed to the events of 9/11 and its aftermath. Details on recruitment methods and Registry findings have been published elsewhere (Brackbill et al., 2009; Farfel et al., 2008; Murphy et al., 2007). In summary, Registry enrollees belong to one or more of five eligibility groups: rescue/recovery workers and volunteers; lower Manhattan residents; area workers; passers-by; and school children and staff. There were two modes of enrollment into the Registry; list-identified enrollees (30\%) were actively recruited from lists provided by governmental agencies, organizations, and employers, while self-identified enrollees (70\%) contacted the Registry via phone or pre-registered on a website in response to intensive outreach efforts (Farfel et al., 2008). The Wave 1 baseline survey (2003-2004) included 68,802 adults, and elicited information on 9/11-related exposures and indicators of physical and mental health (Farfel et al., 2008). The Wave 2 survey (2006-2007) obtained additional information on 9/11-related exposures and provided an update on the health status of 46,602 (68\%) of the original adult enrollees (Brackbill et al., 2009). The Registry protocol was approved by the institutional review boards of the Centers for Disease Control and Prevention and NYC Department of Health and Mental Hygiene.

\subsection{Study sample}

Data on alcohol consumption were collected only at Wave 2; thus, this analysis is limited to adult enrollees (i.e., 18 or older at Wave 1 ) who completed both survey waves $(n=46,374)$. Since a prior history of PTSD is associated with alcohol use (Breslau, 2009; Brewin et al., 2000; Chilcoat and Breslau, 1998; McFarlane, 1998), enrollees with medically diagnosed PTSD prior to 9/11 $(n=602)$, PTSD with a missing date of diagnosis $(n=324)$ or missing information on history of PTSD diagnosis $(n=1449)$ were excluded. Enrollees identifying only as school students or staff ( $n=86$ ) were excluded due to small numbers. Enrollees missing information on binge drinking $(n=2629)$ were also excluded. This resulted in a final sample of 41,284 .

Enrollees completing Wave 2 were more likely to be self-identified, English speakers, male, aged 45-64, non-Hispanic whites, higher income, former smokers, and rescue and recovery workers; however, response rates did not vary by $9 / 11$ exposures (Brackbill et al., 2009).

\subsection{Measures}

2.3.1. Primary outcome-frequent binge drinking. The study outcome, self-reported frequent binge drinking in the last 30 days, was derived from responses to the following question, "Considering all types of alcoholic beverages, how many times during the last 30 days did you have five or more drinks on one occasion?" Enrollees consuming five or more drinks on a single occasion on five or more days in the past
30 days were categorized as frequent binge drinkers (Substance Abuse and Mental Health Services Administration, 2011). This measure of frequent binge drinking has been used in several studies of college students, as it indicates a pattern of high-risk drinking that consists of binge drinking more than once per week and may lead to a greater risk of negative consequences when compared to infrequent binge drinking (Wechsler et al., 2002; White et al., 2006). Enrollees who reported having five or more drinks on a single occasion on fewer than five occasions in the last 30 days or who reported no binge drinking at all were categorized as non-frequent binge drinkers (Substance Abuse and Mental Health Services Administration, 2011).

2.3.2. Sociodemographic variables. We included sociodemographic variables that were associated with excess alcohol use in previous studies: age, gender, race/ethnicity, education, 2002 household income, marital status, and tobacco use; information on these variables was collected at either Wave 1 or 2. Consistent with previous Registry studies (Brackbill et al., 2009; Farfel et al., 2008), enrollees were categorized hierarchically into eligibility groups based on exposure using the following ordering: rescue/recovery worker, lower Manhattan resident, or lower Manhattan area worker/passer-by.

2.3.3. WTC exposures. Exposure to the events of $9 / 11$ was defined with a summary measure shown to be associated with mental health status and unmet mental health care need among Registry enrollees (Brackbill et al., 2013). This measure was defined as the number of positive responses to the following 12 events assessed at either Wave 1 or Wave 2: (1) being in the North or South WTC towers or another collapsed building at the time of the attack; (2) witnessing three or more events (seeing planes hit the buildings, buildings collapsing, people falling or jumping from buildings, people injured, or people running); (3) fear of being injured or killed; (4) having a relative killed on $9 / 11$; (5) having a friend killed on $9 / 11$; (6) having a co-worker killed on $9 / 11$; (7) experiencing intense dust cloud; (8) having lost or damaged possessions: (9) sustaining an injury other than eye irritation/injury; (10) being a rescue/recovery or clean-up worker; (11) having evacuated one's home for at least $24 \mathrm{~h}$ after 9/11; and (12) having lost one's job because of 9/11. Based on the score distribution, exposure was categorized as none/low (0-1 experiences), medium (2-3), high (4-5), and very high (6 or more). This scale covers several dimensions of 9/11-related exposure and each item has been shown to be associated with physical and/or mental health outcomes in previous Registry studies (Brackbill et al., 2009; DiGrande et al., 2011). A similar measure was used by Adams et al. (2006).

2.3.4. Posttraumatic stress disorder. Probable PTSD was assessed at Wave 2 using the PTSD Checklist-Civilian Version (PCL-17). The PCL-17 is a self-reported, 17-item scale corresponding to the criteria in the DSM-IV that is commonly used in epidemiological research (Blanchard et al., 1996; Ruggiero et al., 2003). Selected symptoms were queried as specific to $9 / 11$ and all were current (within the last 30 days). Psychometric properties of the PCL used in this manner in other settings and with other traumatized populations have reported sensitivity ranging from 0.94 to 0.97 , specificity from 0.86 to 0.99 , positive predictive value from 0.70 to 0.97 , and diagnostic efficiency from 0.83 to 0.96 (Blanchard et al., 1996; Ruggiero et al., 2003; Ventureyra et al., 2002; Weathers et al., 1993). Because studies have shown that the diagnostic efficiency of the PCL is improved when using a cut-off of 44 as opposed to 50 (Blanchard et al., 1996), in this study, as well as previous Registry studies (Brackbill et al., 2009; Debchoudhury et al., 2011; Farfel et al., 2008; Li et al., 2011), enrollees with a PCL score of 44 or greater at Wave 2 were considered to have probable PTSD (hereafter referred to as PTSD).

\subsection{Analysis}

Chi-square tests were used to test for significant associations between frequent binge drinking and selected sample descriptors and covariates. Cochran-Armitage tests were used to detect trends between age group and frequent binge drinking and exposure category and frequent binge drinking in the full sample and when stratified by PTSD. Using logistic regression, unadjusted and adjusted odds ratios were calculated to estimate the strength of the association between selected covariates and frequent binge drinking. Based on the results of preliminary analyses adjustment variables were: age group, race/ethnicity, gender, education, 9/11 exposures, tobacco use, and probable PTSD. The final model was stratified by PTSD status. We tested for interaction between PTSD and 9/11 exposures on an additive scale by assessing the relative excess risk due to interaction (RERI) using PROC NLMIXED in SAS (Richardson and Kaufman, 2009). Because there were four exposure categories, we assessed the RERI in three separate models comparing each level of exposure to the no/low exposure group. Analyses were conducted using SAS Version 9.2.

\section{Results}

The majority of the study population was aged $45-64$ years at Wave 2 (50.9\%), non-Hispanic white $(71.5 \%)$, male $(62.1 \%)$, married or living with a partner at Wave $2(69.2 \%)$, and reported having a college or post-graduate education at Wave 1 (54.5\%; Table 1$)$. More than one-fourth (28.2\%) reported a 2002 household income 
Table 1

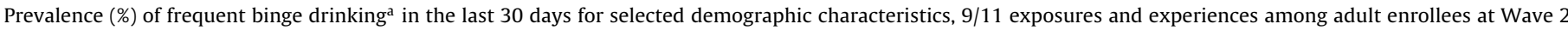
(2006-2007)

\begin{tabular}{|c|c|c|c|c|}
\hline & \multicolumn{4}{|c|}{ Frequent binge drinking in the last 30 days $^{\mathrm{a}}$} \\
\hline & $\begin{array}{l}\text { All subjects } \\
N(\%)\end{array}$ & $\begin{array}{l}\text { Yes } \\
N(\%)\end{array}$ & $\begin{array}{l}\text { No } \\
N(\%)\end{array}$ & $p$-value \\
\hline & $41,284(100.0)$ & $3233(7.8)$ & $38,051(92.2)$ & \\
\hline Age group (years) & & & & $<0.0001^{\mathrm{b}}$ \\
\hline $18-29$ & $2277(5.5)$ & $265(11.6)$ & $2012(88.4)$ & \\
\hline $30-44$ & $15,251(36.9)$ & $1431(9.4)$ & $13,820(90.6)$ & \\
\hline $45-64$ & $20,998(50.9)$ & $1470(7.0)$ & $19,528(93.0)$ & \\
\hline $65+$ & $2758(6.7)$ & $67(2.4)$ & $2691(97.6)$ & \\
\hline Race/ethnicity & & & & $<0.0001$ \\
\hline Non-hispanic white & $29,526(71.5)$ & $2555(8.7)$ & $26,971(91.4)$ & \\
\hline Non-hispanic black & $3941(9.6)$ & $162(4.1)$ & $3779(95.9)$ & \\
\hline Hispanic or latino & $4464(10.8)$ & $356(8.0)$ & $4108(92.0)$ & \\
\hline Multiracial/other & $1340(3.3)$ & $93(6.9)$ & $1247(93.1)$ & \\
\hline Asian & $2013(4.9)$ & $67(3.3)$ & $1946(96.7)$ & \\
\hline Gender & & & & $<0.0001$ \\
\hline Male & $25,643(62.1)$ & $2695(10.5)$ & $22,948(89.5)$ & \\
\hline Female & $15,641(37.9)$ & $538(3.4)$ & $15,103(96.6)$ & \\
\hline Marital status & & & & 0.0006 \\
\hline Married/living with partner & $28,351(69.2)$ & $2146(7.6)$ & $26,205(92.4)$ & \\
\hline Divorced/separated/widowed & $5678(13.9)$ & $440(7.8)$ & $5238(92.3)$ & \\
\hline Never married & $6920(16.9)$ & $620(9.0)$ & $6300(91.0)$ & \\
\hline Education & & & & $<0.0001$ \\
\hline Less than HS & $1153(2.8)$ & $100(8.7)$ & $1053(91.3)$ & \\
\hline HS graduate/GED & 7355(17.9) & $754(10.3)$ & $6601(89.8)$ & \\
\hline Some college & $10,183(24.8)$ & $945(9.3)$ & $9238(90.7)$ & \\
\hline College/post graduate & $22,356(54.5)$ & $1416(6.3)$ & $20,940(93.7)$ & \\
\hline Household income & & & & $<0.0001$ \\
\hline$\geq 100,000$ & $11,641(28.2)$ & $970(8.3)$ & $10,671(91.7)$ & \\
\hline $75,000-99,999$ & $7582(18.4)$ & $645(8.5)$ & 6937(91.5) & \\
\hline$\$ 50,000-74,999$ & $8139(19.7)$ & $698(8.6)$ & $7441(91.4)$ & \\
\hline$\$ 25,000-49,999$ & $7049(17.1)$ & $487(6.9)$ & $6562(93.1)$ & \\
\hline Missing & $3986(9.7)$ & $212(5.3)$ & $3774(94.7)$ & \\
\hline$\leq \$ 24,999$ & $2887(7.0)$ & $221(7.7)$ & $2666(92.3)$ & \\
\hline Eligibility group & & & & $<0.0001$ \\
\hline Rescue and recovery workers & $19,444(47.1)$ & $1903(9.8)$ & $17,541(90.2)$ & \\
\hline Lower Manhattan residents & $5591(13.5)$ & $329(5.9)$ & $5262(94.1)$ & \\
\hline Lower Manhattan area workers and passers-by & $16,249(39.4)$ & $1001(6.2)$ & $15,248(93.8)$ & \\
\hline 9/11 exposure scale & & & & $<0.0001^{\mathrm{b}}$ \\
\hline Very high (6-11 exposures) & $3808(9.2)$ & $523(13.7)$ & $3285(86.3)$ & \\
\hline High ( $4-5$ exposures) & $9665(23.4)$ & $947(9.8)$ & $8718(90.2)$ & \\
\hline Medium ( $2-3$ exposures) & $17,475(42.3)$ & $1305(7.5)$ & $16,170(92.5)$ & \\
\hline None/low (0-1 exposures) & $10,336(25.0)$ & $458(4.4)$ & 9878(95.6) & \\
\hline Tobacco use post- $9 / 11$ at Wave 2 & & & & $<0.0001$ \\
\hline Current & $5561(13.6)$ & $919(16.5)$ & $4642(83.5)$ & \\
\hline Former & $12,565(30.7)$ & $955(7.6)$ & $11,610(92.4)$ & \\
\hline Never & $22,870(55.8)$ & $1333(5.8)$ & $21,537(94.2)$ & \\
\hline Probable PTSD at Wave 2 & & & & $<0.0001$ \\
\hline Yes & $7495(18.7)$ & $1111(14.8)$ & 6384(85.2) & \\
\hline No & $32,696(81.4)$ & $2058(6.3)$ & $30,638(93.7)$ & \\
\hline
\end{tabular}

a 5 or more episodes of binge drinking in the last 30 days.

b $p$-Value for Cochran-Armitage trend test.

of $\$ 100,000$ or greater and the greatest proportion by eligibility group were rescue and recovery workers (47.1\%). Most enrollees reported either a medium (42.3\%) or low $(25.0 \%)$ number of $9 / 11$ exposures. Only $13.6 \%$ were current smokers and more than half (55.8\%) had never smoked and $18.7 \%$ had PTSD.

Overall, $7.8 \%$ of enrollees reported frequent binge drinking in the 30 days prior to the Wave 2 survey. Among frequent binge drinkers, the median number of binge drinking days was 8.0 and the mean was 10.9 ( $\mathrm{SD}=7.0)$. The prevalence of frequent binge drinking decreased with increasing age, with those aged 65 and over the least likely to engage in frequent binge drinking ( $p$-value $<0.0001$; Table 1$)$. The prevalence of frequent binge drinking was higher among non-Hispanic whites, Hispanics, multiracial/other, men, those who were never married, high school graduates, those with an income of $\$ 50,000$ or greater, and current smokers compared to their counterparts ( $p$-values $<0.001$ ). 


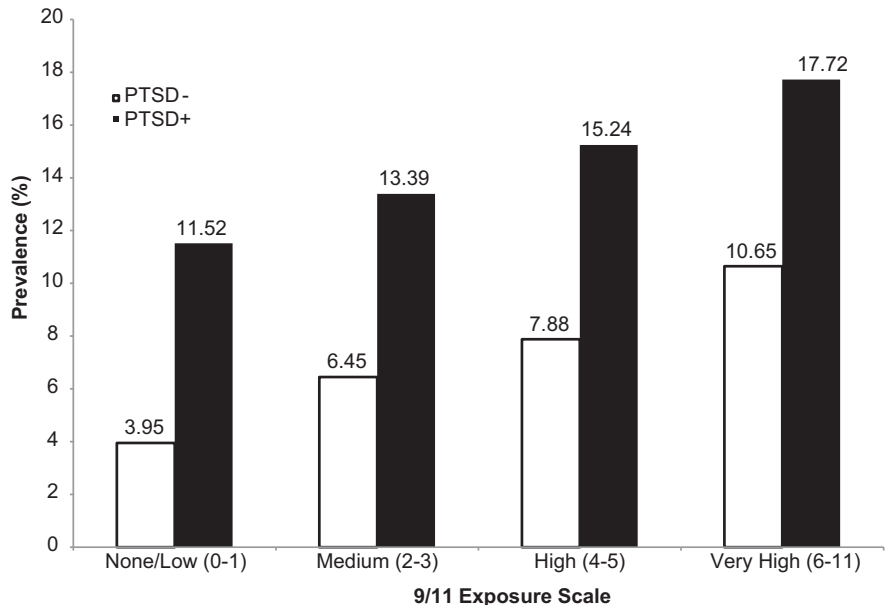

Fig. 1. Prevalence (\%) of frequent binge drinking in the last 30 days by $9 / 11$ exposures and PTSD status among adult enrollees at Wave 2 (2006-2007).

The prevalence of frequent binge drinking was significantly higher among rescue and recovery workers (9.8\%) than lower Manhattan residents (5.9\%) and lower Manhattan area workers/passers-by (6.2\%). Table 1 also shows that the prevalence of frequent binge drinking increased with increased number of 9/11 exposures $(p<0.0001)$. Those with very high and high exposures had a higher prevalence of frequent binge drinking ( $13.7 \%$ and $9.8 \%$, respectively) than those with medium and low exposures ( $7.5 \%$ and $4.4 \%$, respectively). Frequent binge drinking was also significantly elevated among those with PTSD (14.8\%) compared to those without PTSD (6.3\%). As shown in Fig. 1, the prevalence of frequent binge drinking increased with increasing exposure among enrollees with PTSD and without $(p<0.0001)$; the highest prevalence of frequent binge drinking was found among enrollees with PTSD and very high 9/11 exposure (17.7\%).

The $p$-value for the RERI (using relative risk and odds ratio) was not significant for any level of exposure. As such, we concluded that there was not a significant additive interaction between PTSD and number of 9/11 exposures at any level.

Table 2 shows the unadjusted and adjusted odds ratio estimates for the association between frequent binge drinking and demographic characteristics and 9/11 experiences. Although adjusting for selected demographic characteristics and 9/11 experiences changed the magnitude of the observed associations, the overall patterns did not change. Individuals aged 18-29 had a 5.5 times (95\% CI: 4.1-7.3) greater odds of frequent binge drinking compared to those aged 65 years and older; elevated odds were also found for those aged 30-44 and 45-64 (AOR: 3.3; 95\% CI: 2.6-4.3 and AOR: 2.2; 95\% CI: 1.7-2.9). The likelihood of frequent binge drinking was also elevated for males (AOR: 3.1; 95\% CI: 2.8-3.5) compared to females, high school graduates and enrollees with some college (AOR: 1.3 ; 95\% CI: 1.2-1.4 and AOR: $1.2 ; 95 \% \mathrm{CI}: 1.1-1.3$ ) compared to individuals with a college or post-graduate education, and all racial/ethnic groups compared to Asians.

With respect to $9 / 11$ experiences, the odds of frequent binge drinking were more than one and a half times higher among enrollees with high or very high reported 9/11 exposures compared to those with none/low exposures (AOR: 1.6; 95\% CI: 1.4-1.8 and AOR: 1.9 ; 95\% CI: 1.6-2.2, respectively) after adjustment. Current smokers and former smokers were 2.6 (95\% CI: 2.4-2.9) and 1.4 (95\% CI: 1.3-1.5) times more likely to engage in frequent binge drinking than those who had never smoked. Additionally, the odds of frequent binge drinking were more than twice as high among individuals with PTSD than those without (AOR: 2.2; 95\% CI: 2.0-2.4).
Table 2

Odds ratios and 95\% confidence intervals for association between frequent binge drinking ${ }^{\mathrm{a}}$ in the last 30 days and selected demographic characteristics, 9/11 exposures and experiences among adult enrollees at Wave 2 (2006-2007).

\begin{tabular}{|c|c|c|}
\hline & \multirow{2}{*}{\multicolumn{2}{|c|}{$\begin{array}{l}\text { Frequent binge drinking in the last } 30 \\
\text { days }^{\text {a }}\end{array}$}} \\
\hline & & \\
\hline & Unadjusted & Adjusted $^{\mathrm{b}}$ \\
\hline \multicolumn{3}{|l|}{ Age group (years) } \\
\hline $18-29$ & $5.3(4.0-7.0)$ & $5.5(4.1-7.3)$ \\
\hline $30-44$ & $4.2(3.2-5.3)$ & $3.3(2.6-4.3)$ \\
\hline $45-64$ & $3.0(2.4-3.9)$ & $2.2(1.7-2.9)$ \\
\hline $65+$ & 1.00 & 1.00 \\
\hline \multicolumn{3}{|l|}{ Race/ethnicity } \\
\hline Non-hispanic white & $2.8(2.1-3.5)$ & $2.4(1.9-3.1)$ \\
\hline Non-hispanic black & $1.2(0.9-1.7)$ & $1.4(1.0-1.9)$ \\
\hline Hispanic or latino & $2.5(1.9-3.3)$ & $1.9(1.4-2.5)$ \\
\hline Multiracial/other & $2.2(1.6-3.0)$ & $1.8(1.3-2.6)$ \\
\hline Asian & 1.00 & 1.00 \\
\hline \multicolumn{3}{|l|}{ Gender } \\
\hline Male & $3.3(3.0-3.6)$ & $3.1(2.8-3.5)$ \\
\hline Female & 1.00 & 1.00 \\
\hline \multicolumn{3}{|l|}{ Education } \\
\hline Less than HS & $1.4(1.1-1.7)$ & $1.1(0.9-1.4)$ \\
\hline HS graduate/GED & $1.7(1.5-1.9)$ & $1.3(1.2-1.4)$ \\
\hline Some college & $1.5(1.4-1.6)$ & $1.2(1.1-1.3)$ \\
\hline College/post graduate & 1.00 & 1.00 \\
\hline \multicolumn{3}{|l|}{ 9/11 exposure scale } \\
\hline Very high (6-11 exposures) & $3.4(3.0-3.9)$ & $1.9(1.6-2.2)$ \\
\hline High (4-5 exposures) & $2.3(2.1-2.6)$ & $1.6(1.4-1.8)$ \\
\hline Medium (2-3 exposures) & $1.7(1.6-1.9)$ & $1.4(1.2-1.6)$ \\
\hline None/low (0-1 exposures) & 1.00 & 1.00 \\
\hline \multicolumn{3}{|l|}{ Tobacco use post- $9 / 11$ at Wave 2} \\
\hline Current & $3.2(2.9-3.5)$ & $2.6(2.4-2.9)$ \\
\hline Former & $1.3(1.2-1.4)$ & $1.4(1.3-1.5)$ \\
\hline Never & 1.00 & 1.00 \\
\hline \multicolumn{3}{|l|}{ Probable PTSD at Wave 2} \\
\hline Yes & $2.6(2.4-2.8)$ & $2.2(2.0-2.4)$ \\
\hline No & 1.00 & 1.00 \\
\hline
\end{tabular}

a 5 or more episodes of binge drinking in the last 30 days.

b Adjusted for age group, race/ethnicity, gender, education, 9/11 exposures, tobacco use, and probable PTSD.

Table 3 shows the adjusted odds ratio estimates for the association between frequent binge drinking and demographic characteristics stratified by PTSD status. Among enrollees with PTSD we found a marginally significant association between degree of 9/11 exposure and frequent binge drinking, and there was a trend of higher odds with increasing degree of 9/11 exposure. Among enrollees without PTSD, the association between exposure level and frequent binge drinking was significant and was greater than the association found in the unstratified analysis. Specifically, among those without PTSD, the odds of frequent binge drinking were highest for those with high and very high 9/11 exposures (OR: 1.7; $95 \%$ CI: $1.4-1.9$ and OR: 2.1 ; 95\% CI: $1.8-2.5$ ).

\section{Discussion}

Five to six years post-disaster, we found a dose response relationship between the number of 9/11-related exposures and frequent binge drinking. Registry enrollees' likelihood of frequent binge drinking increased significantly with increasing number of 9/11-related exposures. Examining frequent binge drinking enabled us to evaluate a more sustained pattern of binge drinking that cannot be assessed using occasional binge drinking. 
Table 3

Adjusted odds ratios and 95\% confidence intervals for association between frequent binge drinking ${ }^{\mathrm{a}}$ in the last 30 days and selected demographic characteristics, 9/11 exposures and experiences stratified by PTSD among adult enrollees at Wave 2 (2006-2007).

\begin{tabular}{|c|c|c|}
\hline & \multicolumn{2}{|c|}{$\begin{array}{l}\text { Frequent binge drinking in the last } 30 \\
\text { days }^{\text {a }}\end{array}$} \\
\hline & \multicolumn{2}{|l|}{ All subjects } \\
\hline & Probable PTSD ${ }^{\mathrm{b}}$ & No Probable PTSD ${ }^{\mathrm{b}}$ \\
\hline \multicolumn{3}{|l|}{ Age group (years) } \\
\hline $18-29$ & $3.8(2.2-6.6)$ & $6.3(4.5-8.8)$ \\
\hline $30-44$ & $2.7(1.6-4.4)$ & $3.6(2.7-4.9)$ \\
\hline $45-64$ & $2.1(1.3-3.4)$ & $2.3(1.7-3.1)$ \\
\hline $65+$ & 1.00 & 1.00 \\
\hline \multicolumn{3}{|l|}{ Race/ethnicity } \\
\hline Non-hispanic white & $2.1(1.4-3.2)$ & $2.6(1.9-3.6)$ \\
\hline Non-hispanic black & $1.3(0.8-2.2)$ & $1.4(1.0-2.1)$ \\
\hline Hispanic or latino & $2.0(1.3-3.2)$ & $1.8(1.3-2.6)$ \\
\hline Multiracial/other & $1.6(0.9-2.8)$ & $2.0(1.3-3.2)$ \\
\hline Asian & 1.00 & 1.00 \\
\hline \multicolumn{3}{|l|}{ Gender } \\
\hline Male & $2.9(2.5-3.4)$ & $3.3(2.9-3.7)$ \\
\hline Female & 1.00 & 1.00 \\
\hline \multicolumn{3}{|l|}{ Education } \\
\hline Less than HS & $1.1(0.8-1.5)$ & $1.1(0.8-1.6)$ \\
\hline HS graduate/GED & $1.3(1.1-1.5)$ & $1.3(1.1-1.4)$ \\
\hline Some college & $1.1(0.9-1.3)$ & $1.3(1.2-1.4)$ \\
\hline College/post graduate & 1.00 & 1.00 \\
\hline \multicolumn{3}{|l|}{ 9/11 exposure scale } \\
\hline Very high (6-11 exposures) & $1.3(1.0-1.8)$ & $2.1(1.8-2.5)$ \\
\hline High (4-5 exposures) & $1.3(1.0-1.6)$ & $1.7(1.4-1.9)$ \\
\hline Medium (2-3 exposures) & $1.1(0.8-1.4)$ & $1.5(1.3-1.6)$ \\
\hline None/low ( $0-1$ exposures) & 1.00 & 1.00 \\
\hline \multicolumn{3}{|l|}{ Tobacco use post- $9 / 11$ at Wave 2} \\
\hline Current & $1.7(1.5-2.0)$ & $3.3(2.9-3.7)$ \\
\hline Former & $1.1(0.9-1.3)$ & $1.5(1.4-1.7)$ \\
\hline Never & 1.00 & 1.00 \\
\hline
\end{tabular}

a 5 or more episodes of binge drinking in the last 30 days.

b Adjusted for age group, race/ethnicity, gender, education, 9/11 exposures, and tobacco use.

Registry enrollees had a higher prevalence of frequent binge drinking (7.8\%) when compared to both NYC adults (5.7\%; New York City Department of Health and Mental Hygiene, 2007) and adults nationally (6.7\%; Substance Abuse and Mental Health Services Administration, 2011). Similar to findings from one to two years after 9/11 (Boscarino et al., 2006), we found elevated odds of frequent binge drinking among 18-29 year olds, males, non-Hispanic whites, Hispanics, multiracial/other, and those with a high school diploma or equivalent. Additionally, smokers and individuals with probable PTSD had an increased likelihood of frequent binge drinking.

We also observed a strong association between increasing intensity of 9/11 exposures and frequent binge drinking. Consistent with our results, a previous study of a 9/11-exposed population (Boscarino et al., 2006) demonstrated elevated levels of binge drinking among individuals highly and very highly exposed to the WTC disaster one and two years post-9/11 even after adjustment for PTSD; however, the association between PTSD and binge drinking was not significant as it was in our study. In the present study, both PTSD and level of 9/11 exposure were significantly associated with frequent binge drinking. Moreover, 9/11 exposure was associated with frequent binge drinking irrespective of PTSD status. This difference may be attributable to the lower prevalence of PTSD among participants in Boscarino's study compared to ours, as well as to the different measures of binge drinking used in the two studies (any binge drinking in the prior year versus five episodes of binge drinking in the last 30 days).

\subsection{Strengths and limitations}

This study has several limitations. The Wave 2 survey was completed by $68 \%$ of the original adult sample. Those not completing Wave 2 were more likely to have had PTSD at Wave 1 . We do not know if frequent binge drinkers were more or less likely to complete Wave 2, as alcohol consumption was not assessed at Wave 1 . However, September $11^{\text {th }}$ experiences were not associated with Wave 2 survey completion (Brackbill et al., 2009). All data including alcohol intake were self-reported, which can under-represent actual consumption (Gordon and Kannel, 1984; Midanik, 1988; Stahre et al., 2006). Additionally, alcohol use was assessed only at Wave 2 and does not include information about enrollees' prior drinking habits, post-disaster changes in consumption, or other alcohol-related outcomes. Enrollees missing binge drinking information were excluded from our analysis; this may have biased results, as these enrollees were more likely to be older, female, black, Hispanic, Asian, divorced, residents, never smokers, listidentified, to have lower income, lower education, and low 9/11 exposures and less likely to have PTSD. We excluded a relatively small number of enrollees $(n=602)$ with a history of medically diagnosed PTSD prior to 9/11 (pre-9/11 PTSD) to improve clarity of interpretation, since their inclusion would add complicating issues such as resensitization to trauma cues. However, the prevalence of frequent binge drinking among those with pre-9/11 PTSD was not significantly different from those without and their inclusion in our final models did not modify our findings. Data on 9/11 exposures were self-reported and were collected two to three years after the event and therefore may be subject to recall bias.

Among the strengths of this analysis are its large sample size and the range of data on sociodemographic characteristics and 9/11 exposures. In contrast to other studies examining post-9/11 alcohol use, Registry enrollees represent a directly exposed population with varying degrees of exposure and comprise individuals who lived or worked in close proximity to the WTC site and/or participated in rescue, recovery, and clean-up activities. While the Registry is not necessarily representative of either NYC or the entire exposed population, results from analyses of these data are still informative about the experiences of populations directly exposed to large-scale traumatic events. Moreover, Registry findings have been consistent with other 9/11 cohorts (e.g., WTC Health Program for Responders; Pietrzak et al., 2014; Stellman et al., 2008, and the Fire Department of New York; Berninger et al., 2010) as well as the broader literature on post-disaster physical health, traumatic stress and other mental health outcomes. In addition, we checked for possible selection bias by adjusting for mode of enrollment in the Registry (list- versus self-identified); however we did not maintain this variable in our final model due to lack of statistical significance. Finally, Registry data used in this study were obtained five to six years after $9 / 11$; this time point represents the furthest observation point on post-9/11 alcohol use to date.

\section{Conclusion}

Understanding the effects of traumatic exposure on alcohol use among directly exposed adults is important and can help to identify factors that increase the risk of alcohol misuse following a disaster, better inform disaster policy and response, and improve the provision of psychological and alcohol screening and counseling in the aftermath of a large-scale traumatic event. Our findings suggest that 9/11 exposure had an impact on frequent binge drinking five 
to six years after the event among Registry enrollees whether or not they had PTSD. Future studies will follow participants longitudinally in order to investigate the long-term impacts of exposure and course of alcohol use disorders in the 9/11-exposed population and across racial or ethnic groups.

\section{Role of the funding source}

This study was supported by the National Institute for Occupational Safety and Health (cooperative agreement numbers 5U50/OH009739 and 1E11/OH009630), and the Agency for Toxic Substances and Disease Registry (ATSDR) which included support from CDC-NCEH the National Center for Environmental Health (cooperative agreement number U50/ATU272750), of the Centers for Disease Control and Prevention (A.E.W., K.C., C.B.M., J.E.C., M.R.F., S.D.S.), the National Institutes of Health (K.M.K., grant number K01AA021511 and D.S.H. grant number K05AA014223), the New York State Psychiatric Institute (D.H.) and by the New York City Department of Health and Mental Hygiene (A.E.W., K.C., C.B.M., J.E.C., M.R.F., S.D.S.). Its contents are solely the responsibility of the authors and do not necessarily represent the official views of CDC.

\section{Contributors}

Dr. Welch and Ms. Caramanica designed the study, managed the literature searches, performed the statistical analyses and wrote the first draft of the manuscript. Drs. Maslow, Keyes, Stellman and Hasin contributed to the study design and analyses. All authors contributed to data interpretation and to the revision of the manuscript. All authors have approved the final manuscript.

\section{Conflict of interest}

All authors declare that they have no conflicts of interest.

\section{Acknowledgements}

We are grateful for the helpful advice and comments from Carolyn Greene, Jim Hadler, Hillary Kunins, and Denise Paone.

\section{References}

Adams, R.E., Boscarino, J.A., 2005. Stress and well-being in the aftermath of the World Trade Center attack: the continuing effects of a communitywide disaster. J. Community Psychol. 33, 175-190.

Adams, R.E., Boscarino, J.A., Galea, S., 2006. Alcohol use, mental health status and psychological well-being 2 years after the World Trade Center attacks in New York City. Am. J. Drug Alcohol Abuse 32, 203-224.

Berninger, A., Webber, M.P., Cohen, H.W., Gustave, J., Lee, R., Niles, J.K., Chiu, S. Zeig-Owens, R., Soo, J., Kelly, K., Prezant, D.J., 2010. Trends of elevated PTSD risk in firefighters exposed to the World Trade Center disaster: 2001-2005. Public Health Rep. 125, 556-566.

Blanchard, E.B., Jones-Alexander, J., Buckley, T.C., Forneris, C.A., 1996. Psychometric properties of the PTSD Checklist (PCL). Behav. Res. Ther. 34, 669-673.

Boscarino, J.A., Adams, R.E., Galea, S., 2006. Alcohol use in New York after the terrorist attacks: a study of the effects of psychological trauma on drinking behavior. Addict. Behav. 31, 606-621.

Boscarino, J.A., Kirchner, H.L., Hoffman, S.N., Sartorius, J., Adams, R.E., 2011. PTSD and alcohol use after the World Trade Center attacks: a longitudinal study. J. Trauma Stress 24, 515-525.

Brackbill, R.M., Hadler, J.L., DiGrande, L., Ekenga, C.C., Farfel, M.R., Friedman, S., Perlman, S.E., Stellman, S.D., Walker, D.J., Wu, D., Yu, S., Thorpe, L.E., 2009. Asthma and posttraumatic stress symptoms 5 to 6 years following exposure to the World Trade Center terrorist attack. JAMA 302, 502-516.

Brackbill, R.M., Stellman, S.D., Perlman, S.E., Walker, D.J., Farfel, M.R., 2013. Mental health of those directly exposed to the World Trade Center disaster: unmet mental health care need, mental health treatment service use, and quality of life. Soc. Sci. Med. 81, 110-114.

Breslau, N., 2009. The epidemiology of trauma PTSD, and other posttrauma disorders. Trauma Violence Abuse 10, 198-210.

Brewin, C.R., Andrews, B., Valentine, J.D., 2000. Meta-analysis of risk factors for posttraumatic stress disorder in trauma-exposed adults. J. Consult. Clin. Psychol. 68, 748-766.
Chilcoat, H.D., Breslau, N., 1998. Investigations of causal pathways between PTSD and drug use disorders. Addict. Behav. 23, 827-840.

Debchoudhury, I., Welch, A.E., Fairclough, M.A., Cone, J.E., Brackbill, R.M., Stellman, S.D., Farfel, M.R., 2011. Comparison of health outcomes among affiliated and lay disaster volunteers enrolled in the World Trade Center Health Registry. Prev. Med. 53, 359-363.

DiGrande, L., Neria, Y., Brackbill, R.M., Pulliam, P., Galea, S., 2011. Long-term posttraumatic stress symptoms among 3,271 civilian survivors of the September 11, 2001, terrorist attacks on the World Trade Center. Am. J. Epidemiol. 173, 271-281.

DiMaggio, C., Galea, S., Li, G., 2009. Substance use and misuse in the aftermath of terrorism. A Bayesian meta-analysis. Addiction 104, 894-904.

Farfel, M., DiGrande, L., Brackbill, R., Prann, A., Cone, J., Friedman, S., Walker, D.J., Pezeshki, G., Thomas, P., Galea, S., Williamson, D., Frieden, T.R., Thorpe, L., 2008. An overview of 9/11 experiences and respiratory and mental health conditions among World Trade Center Health Registry enrollees. J. Urban Health 85, 880-909.

Flory, K., Hankin, B.L., Kloos, B., Cheely, C., Turecki, G., 2009. Alcohol and cigarette use and misuse among Hurricane Katrina survivors: psychosocial risk and protective factors. Subst. Use Misuse 44, 1711-1724.

Gordon, T., Kannel, W.B., 1984. Drinking and mortality The Framingham Study. Am. J. Epidemiol. 120, 97-107.

Grieger, T.A., Fullerton, C.S., Ursano, R.J., 2003. Posttraumatic stress disorder, alcoho use, and perceived safety after the terrorist attack on the pentagon. Psychiatr. Serv. 54, 1380-1382.

Hasin, D.S., Keyes, K.M., Hatzenbuehler, M.L., Aharonovich, E.A., Alderson, D., 2007 Alcohol consumption and posttraumatic stress after exposure to terrorism: effects of proximity, loss, and psychiatric history. Am. J. Public Health 97 2268-2275.

Keyes, K.M., Hatzenbuehler, M.L., Hasin, D.S., 2011. Stressful life experiences, alcoho consumption, and alcohol use disorders: the epidemiologic evidence for four main types of stressors. Psychopharmacology (Berlin) 218, 1-17.

Li, J., Brackbill, R.M., Stellman, S.D., Farfel, M.R., Miller-Archie, S.A., Friedman, S. Walker, D.J., Thorpe, L.E., Cone, J., 2011. Gastroesophageal reflux symptoms and comorbid asthma and posttraumatic stress disorder following the 9/11 terrorist attacks on World Trade Center in New York City. Am. J. Gastroenterol. 106, 1933-1941.

McFarlane, A.C., 1998. Epidemiological evidence about the relationship between PTSD and alcohol abuse: the nature of the association. Addict. Behav. 23, 813-825.

Midanik, L.T., 1988. Validity of self-reported alcohol use: a literature review and assessment. Br. J. Addict. 83, 1019-1030.

Murphy, J., Brackbill, R.M., Thalji, L., Dolan, M., Pulliam, P., Walker, D.J., 2007. Measuring and maximizing coverage in the World Trade Center Health Registry. Stat. Med. 26, 1688-1701.

North, C.S., Tivis, L., McMillen, J.C., Pfefferbaum, B., Spitznagel, E.L., Cox, J., Nixon, S Bunch, K.P., Smith, E.M., 2002. Psychiatric disorders in rescue workers after the Oklahoma City bombing. Am. J. Psychiatry 159, 857-859.

New York City Department of Health and Mental Hygiene, 2007. Epiquery: NYC Interactive Health Data System-Community Health Survey 2007. New York City Department of Health and Mental Hygiene (https://a816-healthpsi. nyc.gov/epiquery/EpiQuery/). Accessed 4 February 2013.

Pietrzak, R.H., Feder, A., Singh, R., Schechter, C.B., Bromet, E.J., Katz, C.L., Reissman, D.B., Ozbay, F., Sharma, V., Crane, M., Harrison, D., Herbert, R., Levin, S.M., Luft, B.J., Moline, J.M., Stellman, J.M., Udasin, I.G., Landrigan, P.J., Southwick, S.M., 2014. Trajectories of PTSD risk and resilience in World Trade Center responders: an 8-year prospective cohort study. Psychol. Med. 44, 205-219.

Richardson, D.B., Kaufman, J.S., 2009. Estimation of the relative excess risk due to interaction and associated confidence bounds. Am. J. Epidemiol. 169, 756-760.

Ruggiero, K.J., Del Ben, K., Scotti, J.R., Rabalais, A.E., 2003. Psychometric properties of the PTSD Checklist-Civilian Version. J. Trauma Stress 16, 495-502.

Stellman, J.M., Smith, R.P., Katz, C.L., Sharma, V., Charney, D.S., Herbert, R., Moline J., Luft, B.J., Markowitz, S., Udasin, I., Harrison, D., Baron, S., Landrigan, P.J., Levin, S.M., Southwick, S., 2008. Enduring mental health morbidity and social function impairment in world trade center rescue, recovery, and cleanup workers: the psychological dimension of an environmental health disaster. Environ. Health Perspect. 116, 1248-1253.

Substance Abuse and Mental Health Services Administration, 2011. Results from the 2010 National Survey on Drug Use and Health: Summary of National findings. Substance Abuse and Mental Health Services Administration (http://www.samhsa.gov/data/NSDUH/2k10NSDUH/2k10Results.htm\#3.1). Accessed 4 February 2013.

Stahre, M., Naimi, T., Brewer, R., Holt, J., 2006. Measuring average alcohol consumption: the impact of including binge drinks in quantity-frequency calculations. Addiction 101, 1711-1718.

Ventureyra, V.A., Yao, S.N., Cottraux, J., Note, I., De Mey-Guillard, C., 2002. The validation of the Posttraumatic Stress Disorder Checklist Scale in posttraumatic stress disorder and nonclinical subjects. Psychother. Psychosom. 71, 47-53.

Vlahov, D., Galea, S., Ahern, J., Resnick, H., Boscarino, J.A., Gold, J., Bucuvalas, M., Kilpatrick, D., 2004. Consumption of cigarettes, alcohol, and marijuana among New York City residents six months after the September 11 terrorist attacks. Am. J. Drug Alcohol Abuse 30, 385-407.

Vlahov, D., Galea, S., Ahern, J., Rudenstine, S., Resnick, H., Kilpatrick, D., Crum R.M., 2006. Alcohol drinking problems among New York City residents after the September 11 terrorist attacks. Subst. Use Misuse 41, 1295-1311. 
Vlahov, D., Galea, S., Resnick, H., Ahern, J., Boscarino, J.A., Bucuvalas, M., Gold, J., Kilpatrick, D., 2002. Increased use of cigarettes, alcohol, and marijuana among Manhattan, New York, residents after the September 11th terrorist attacks. Am. J. Epidemiol. 155, 988-996.

Weathers, F., Litz, B., Herman, D., Huska, J., Keane, T., 1993. The PTSD Checklist (PCL): reliability, validity, and diagnostic utility. In: Paper presented at the Annual Convention of the International Society for Traumatic Stress Studies, San Antonio,
Wechsler, H., Lee, J.E., Kuo, M., Seibring, M., Nelson, T.F., Lee, H., 2002. Trends in college binge drinking during a period of increased prevention efforts. Findings from 4 Harvard School of Public Health College Alcohol Study surveys: 1993-2001. J. Am. College Health 50, 203-217.

White, A.M., Kraus, C.L., Swartzwelder, H., 2006. Many college freshmen drink at levels far beyond the binge threshold. Alcohol. Clin. Exp. Res. 30, 1006-1010. 